\title{
Triaxially deformed sodium clusters in a self-consistent microscopic description
}

\author{
G. Lauritsch, P.-G. Reinhard \\ Institut für Theoretische Physik, Universität Erlangen, Erlangen, Germany \\ J. Meyer \\ Institut de Physique Nucleaire, Université Lyon-I, Lyon, France
}

and

M. Brack

Institut für Theoretische Physik, Universität Regensburg, Regensburg, Germany

Received 17 June 1991; revised manuscript received 11 September 1991; accepted for publication 18 September 1991

Communicated by B. Fricke

\begin{abstract}
We have performed self-consistent Kohn-Sham calculations for the triaxially deformed sodium clusters $\mathrm{Na}_{12}$ and $\mathrm{Na}_{14}$. We use the jellium approach and compute the properties of the valence electrons with an exchange-correlation energy functional in the local density approximation. In both clusters a pronounced shape isomerism is obtained. We find a well-developed triaxial groundstate deformation for $\mathrm{Na}_{12}$ which produces a triple-peaked dipole surface plasmon, as experimentally observed, whereas $\mathrm{Na}_{14}$ maintains axial symmetry but with two almost degenerate oblate and prolate minima.
\end{abstract}

Recent photoabsorption measurements on free sodium clusters [1] have revealed a multipeak structure of the absorption cross section which can be attributed to surface plasmon resonances in deformed systems. In fact, both the phenomenological ellipsoidal shell model of Clemenger [2] and self-consistent Kohn-Sham calculations [3] with imposed axial symmetry for the valence electron system in the jellium approach have revealed that sodium clusters $\mathrm{Na}_{N}$ with atomic numbers $8<N<20$ and $20<N<40$ may be deformed in their ground states. Depending on whether the shape is axial or triaxial, one therefore can expect a splitting of the dipole plasmon resonance into two or three peaks, respectively, corresponding to collective vibrations of the valence electrons in the directions of the principle axes. Indeed, the splittings found in ref. [1] for the clusters with $N=9,10$ and 12 are in fair qualitative agreement with the axis ratios of the corresponding ellipsoidal deformations predicted by the Clemenger model [2]. In particular, the predicted triaxial shape of $\mathrm{Na}_{12}$ has been confirmed by a triple-peaked structure of the cross section.

In this note we present fully self-consistent microscopic calculations for triaxially deformed sodium clusters. We have solved the Kohn-Sham equations for the valence electrons of the clusters $\mathrm{Na}_{12}$ and $\mathrm{Na}_{14}$, treating the ionic background in the jellium approximation [3-5]. We use the same energy density functional as Ekardt and Penzar [3,5] which includes exchange and correlation energies in the local density approximation. In contrast to these authors, we use a jellium density with a diffuse surface profile. This modified jellium model has been shown $[6,7]$ to lead to a better fit of experimental dipole polarisabilities and plasmon surface resonances in spherical clusters than is the case for the standard model with a sharp-edged jellium density [8-10]. We describe the spherical jellium density $\rho_{\mathrm{J}}(r)$ by a Fermi function with a surface width of 1.0 a.u. and 
a half-density radius adjusted such that $\rho_{\mathrm{J}}(0)$ has the value corresponding to a Wigner-Seitz radius $r_{\mathrm{s}}=$ 3.96 a.u. [7]. For deformed clusters, the Fermi function is scaled by the transformation $\rho_{\mathrm{J}}(x, y, z) \rightarrow$ $\rho_{\mathrm{J}}\left(\eta_{x} x, \eta_{y} y, \eta_{z} z\right)$, where $\eta_{x}=\eta_{y}=\eta_{z}^{-2}$ produces an axially symmetric quadrupole deformation and $\eta_{x} \neq \eta_{y}$ generates triaxial shapes. Volume conservation is maintained by $\eta_{x} \eta_{y} \eta_{z}=1$.

In ref. [3] the total energy of the cluster was minimized with respect to axial quadrupole deformations. The idea behind this approach is to assume that the ionic background is very "ductile" and adapts itself to the shape preferred by the valence electrons; this assumption has lead to the successful prediction [5] of the magic numbers $N=8,20,40$, $58,92, \ldots$ of spherical clusters. In the present work we do not only want to find the (eventually triaxial) deformed ground-state deformation of a given cluster, but to exhibit the shape isomerism by presenting explicitly the total energy as a function of the deformations given to the jellium background. We therefore perform the Kohn-Sham calculations on a series of fixed points in deformation space and exhibit the results in the form of potential energy surfaces (PES). Since triaxial deformations require rather elaborate three-dimensional calculations, we have also developed a code with imposed axial symmetry; we shall present and compare results of both codes in this paper.

Our three-dimensional (3D) code makes use of a mixed coordinate- and momentum-space representation connected by the fast Fourier transformation technique. The kinetic energy is evaluated in momentum space whereas the potential acts in coordinate space. That has been found to be the optimum strategy for saturating systems of this size [11]. We employ spin-symmetry, occupying each spatial single-particle state twice, and assume reflection symmetry in $x$ - and $y$-direction. The Coulomb problem is solved using the Fourier representation of the Laplacian operator [12]. The efficiency of the Fourier representation allows to use a grid $N_{x} \times N_{y} \times N_{z}=32 \times 32 \times 64$ points with a spatial grid size of $\Delta x=\Delta y=\Delta z=0.9$ a.u. The Kohn-Sham equations are solved with the damped gradient iteration method $[11,13]$. The average total CPU time on a CRAY Y/MP for one deformation point in $\mathrm{Na}_{14}$ is $30 \mathrm{~s}$.
The reflection symmetry in $x$ - and $y$-direction defines the parities $\Pi_{x}$ and $\Pi_{y}$. We therefore have to consider various competing single-particle configurations for each cluster. For both $\mathrm{Na}_{12}$ and $\mathrm{Na}_{14}$, we fill the four lowest states (giving $N=8$ ) with the sequence $\left(\Pi_{x}, \Pi_{y}\right)=(+,+),(-,+),(+,-),(+,+)$, each state having two spin directions. The further filling differs. We list in table 1 the energetically most favorable configurations. Each of them produces its own PES with a stable minimum. The lowest minimum determines the stable ground-state configuration, whereas the other minima correspond to isomeric states.

The triaxial shapes of the deformed jellium density are most conveniently classified in terms of the Hill-Wheeler coordinates $(\beta, \gamma)$ for quadrupole deformations [14]. $\beta$ describes the overall quadrupole deformation. $\gamma=0^{\circ}, 120^{\circ}, 240^{\circ}$ describe prolate and $\gamma=60^{\circ}, 180^{\circ}, 300^{\circ}$ oblate deformations; all other $\gamma$ give truly triaxial shapes. There is the usual symmetry in the $(\beta, \gamma)$ plane: the PES is fully determined if it is known in the first sextant $0 \leqslant \gamma \leqslant 60^{\circ}$. This holds only if all possible electronic configurations (i.e. parities in the occupied states) are considered. The PES for one fixed configuration is possibly different in the first three sextants $0 \leqslant \gamma \leqslant 180^{\circ}$ (see fig. 1). For $\gamma=0^{\circ}$, the often used ratio $q$ of the major axes is related to the deformation $\beta$ by

$q=\sqrt{\frac{1+\sqrt{5 / \pi} \beta}{1-\sqrt{5 / 4 \pi} \beta}}$.

For first explorations and systematic investigations, it is more economical to perform a Kohn-Sham

Table 1

Selection of parity quantum numbers for the electron states above $N=8$ for different configurations in $\mathrm{Na}_{12}$ and $\mathrm{Na}_{14}$.

\begin{tabular}{llll}
\hline \multirow{2}{*}{ Name } & \multicolumn{4}{l}{$\left(\Pi_{x}, \Pi_{y}\right)$ of state no. } & 7 \\
& 5 & 6 & \\
\hline $\mathrm{Na}_{12}$ triaxial & $(+,+)$ & $(-,-)$ & \\
$\mathrm{Na}_{12}$ prolate & $(+,-)$ & $(-,+)$ & \\
$\mathrm{Na}_{12}$ oblate & $(+,+)$ & $(+,+)$ & \\
$\mathrm{Na}_{14}$ prolate & $(+,+)$ & $(+,-)$ & $(-,+)$ \\
$\mathrm{Na}_{14}$ oblate & $(+,+)$ & $(+,+)$ & $(-,-)$ \\
$\mathrm{Na}_{14}$ spher. I & $(-,-)$ & $(-,+)$ & $(+,-)$ \\
$\mathrm{Na}_{14}$ spher. II & $(+,+)$ & $(+,+)$ & $(+,+)$ \\
\hline
\end{tabular}




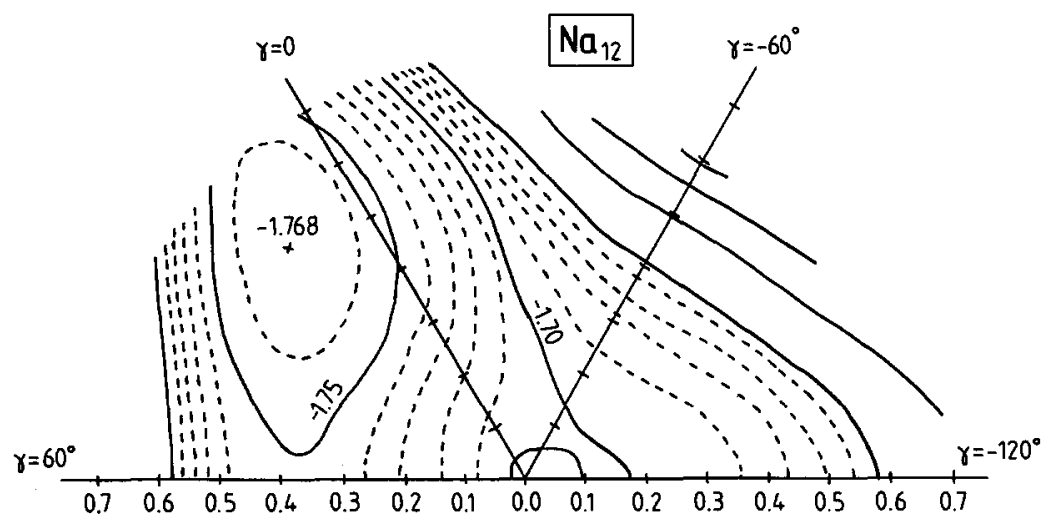

Fig. 1. Potential energy surface in steps of $0.01 \mathrm{Ry}$ (full lines every $0.05 \mathrm{Ry}$ ) in the $(\beta, \gamma)$ plane for $\mathrm{Na}_{12}$ in the triaxial configuration (see table 1). The triaxial minimum with $\beta=0.54, \gamma=15^{\circ}$ has an energy of $-1.768 \mathrm{Ry}$.

calculation with imposed axial symmetry and then to use the triaxial code to check whether the axial minima are stable against triaxial deformations. We have therefore developed a two-dimensional (2D) axial code, adapting a method used in nuclear physics [15]. Hereby the Kohn-Sham equations are solved by diagonalizing the total potential in an axially deformed harmonic oscillator basis using cylindrical coordinates; the electrostatic (direct) Coulomb potential is obtained in each iteration by straightforward integration. For the clusters investigated here, we truncate the oscillator basis at a constant energy corresponding to the inclusion of 12 major shells at sphericity (see ref. [15] for the technical details). We have ensured that the results are stationary with respect to changes of the bases parameters. The integrations are done by Gauss-Hermite and Gauss-Laguerre quadrature in the two respective spatial coordinates, using $32 \times 32$ mesh points.

Fig. 1 exhibits the PES for $\mathrm{Na}_{12}$ resulting from our 3D calculations for the energetically most favorable configuration, listed as "triaxial" in table 1. The ground state at $E=-1.768 \mathrm{Ry}$ is clearly triaxial with the deformation $\beta=0.54, \gamma=15^{\circ}$ and is energetically well separated from the competing "prolate" and "oblate" configurations (see fig. 3). This triaxial deformation compares favourably with the result $\beta=0.54, \gamma=19^{\circ}$ of the phenomenological shell model [2]. For $\mathrm{Na}_{14}$, the 3D calculations yield axially symmetric minima in all the configurations listed in table 1 . We show in fig. 2 the PES in the $(\beta, \gamma)$ plane

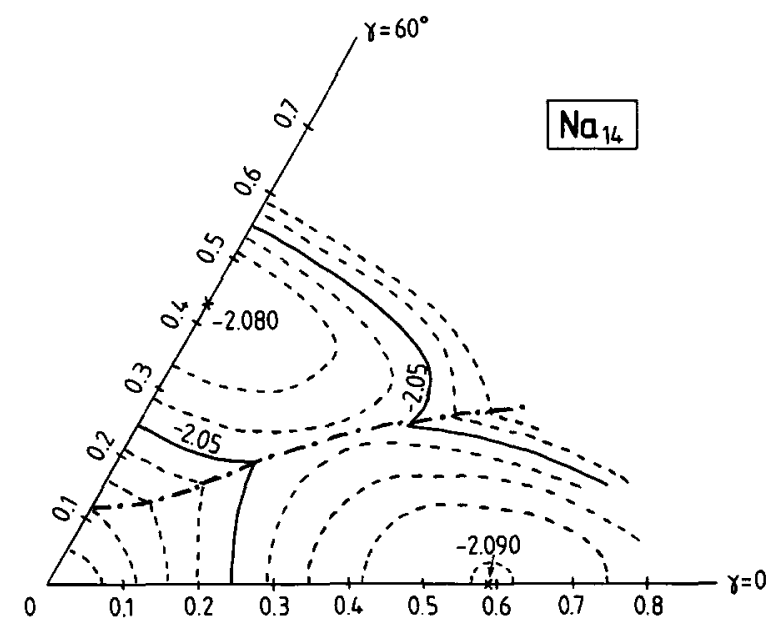

Fig. 2. Same as fig. 1, but for $\mathrm{Na}_{14}$ (prolate and oblate configurations as listed in table 1 ). The thick dash-dotted line indicates the line where the two surfaces cut each other.

for the two lowest configurations ("prolate" and "oblate"). Note that the two minima are energetically almost degenerate. The oblate minimum is rather soft in the $\gamma$-direction whereas the prolate minimum seems to predict stiffer $\gamma$-vibrations.

In fig. 3 we show cuts of the PES along the axes $\gamma=0^{\circ}(\beta \geqslant 0)$ and $\gamma=60^{\circ}(\beta \leqslant 0)$ versus the quadrupole deformation $\beta$. The agreement in the energies between the $2 \mathrm{D}$ and the $3 \mathrm{D}$ results, within a few parts per thousand, lies within the limits of the estimated numerical accuracy of either code and is therefore very rewarding. We see that the prolate and oblate 


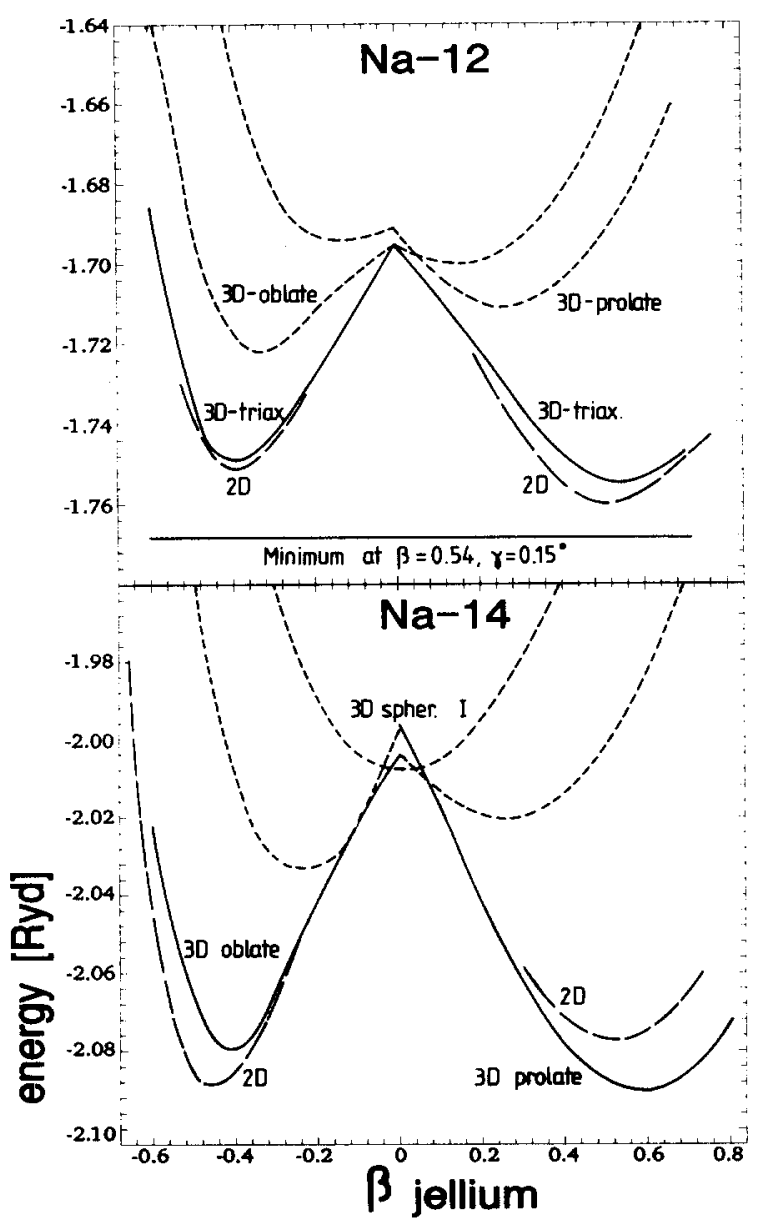

Fig. 3. Deformation energy (in Ry) for axially symmetric deformations of $\mathrm{Na}_{12}$ (upper part) and $\mathrm{Na}_{14}$ (lower part), shown versus the quadrupole deformation $\beta$. (2D results: dashed lines. Cuts of 3D results: full lines (lowest configuration) and dotted lines (higher configurations); single-particle configurations named as in table 1.) The energy of the triaxial $\mathrm{Na}_{12}$ ground state is indicated by a full horizontal line.

minima of $\mathrm{Na}_{12}$, too, are nearly degenerate. The axis ratios $q$ at the axially symmetric minima agree fairly well with the published values of both the phenomenological shell model [2] and the sharp-edged spheroidal jellium model [3]. For instance, for the oblate minimum in $\mathrm{Na}_{14}$ we find $q=0.57$ (2D) or $q=0.60$ (3D), compared with $q=57$ in ref. [3] and $q=0.53$ in ref. [2].

The cusps in the PES at spherical symmetry are unphysical. The residual interaction between the different electronic configurations will smooth out these cusps and thereby lower the barriers separating the isomeric minima (cf. the Jahn-Teller effect [16]). Moreover, the free variation of higher multipole deformations of the jellium may further modify somewhat the relative positions of the stationary points in the PES.

The pronounced shape isomerism found here for both clusters bears a certain resemblance to that found in the totally different approach of quantum chemistry, where the clusters are described fully microscopically in ab initio calculations using the BornOppenheimer approximation for the relative positions of the atoms [17]. Clearly, the latter approach is a priori more adequate for the description of microclusters. But our results seem to indicate that a full exploitation of the three-dimensional deformation degrees of freedom in the jellium model will lead to a partial reconciliation of these two otherwise quite orthogonal approaches. However, a quantitative comparison exceeds the limits of this Letter and will the object of future studies.

We have finally investigated the resonance energies of collective dipole oscillations of the valence electrons against the ionic background. As a first estimate, we have approximated them as pure surface plasmons (i.e., purely translational dipole modes) by the random phase approximation (RPA) sum rule expression $[10,18] \hbar \omega=\left[m_{3} \text { (D) } / m_{1}(\mathbf{D})\right]^{1 / 2}$, where $\mathrm{D}$ is the dipole operator and $m_{k}$ is the $k$ th energyweighted moment of the RPA dipole strength function. For deformed clusters, the expression found in ref. [10] generalises trivially to

$\left(\hbar \omega_{i}\right)^{2}=\frac{\hbar^{2}}{N m} \int \mathrm{d}^{3} r \rho_{\mathrm{el}}(\boldsymbol{r}) \frac{\partial^{2}}{\partial r_{i}^{2}} V_{\mathrm{J}}(\boldsymbol{r})$,

where $\rho_{\mathrm{el}}(\boldsymbol{r})$ is the electron density, $V_{\mathrm{J}}(\boldsymbol{r})$ the electrostatic jellium potential, and $i$ runs over the spatial directions, i.e., $r_{i} \in\{x, z, y\}$ for the triaxial case and $r_{i} \in\{r, z\}$ for the axial case. We have evaluated the resonance energies $\hbar \omega_{i}$ for the most prominant minima in both clusters. The results are shown in table 2. We find three different energies for $\mathrm{Na}_{12}$, related to its triaxial shape in the ground state. It is most interesting to compare them with the experimental data of ref. [1] quoting peaks at $\hbar \omega_{i}=0.156,0.182$, and $0.203 \mathrm{Ry}$. Our results are too high in energy by about $10-15 \%$ due to the simple dipole sum-rule approx- 
Table 2

Dipole surface plasmon energies (in Ry) in the RPA dipole sumrule approximation (1). The numbers in the upper row are from the $3 \mathrm{D}$ code, those in the lower row from the axial 2D code. (Note the perfect agreement.)

\begin{tabular}{|c|c|c|c|c|c|c|}
\hline \multirow{2}{*}{\multicolumn{3}{|c|}{$\begin{array}{l}\mathrm{Na}_{12} \\
\text { triaxial }\end{array}$}} & \multicolumn{4}{|l|}{$\mathrm{Na}_{14}$} \\
\hline & & & \multicolumn{2}{|l|}{ oblate } & \multicolumn{2}{|l|}{ prolate } \\
\hline$\hbar \omega_{x}$ & $\hbar \omega_{y}$ & $\hbar \omega_{z}$ & $\hbar \omega_{r}$ & $\hbar \omega_{z}$ & $\hbar \omega_{r}$ & $\hbar \omega_{z}$ \\
\hline 0.211 & 0.239 & 0.171 & $\begin{array}{l}0.187 \\
0.188\end{array}$ & $\begin{array}{l}0.251 \\
0.252\end{array}$ & $\begin{array}{l}0.229 \\
0.229\end{array}$ & $\begin{array}{l}0.171 \\
0.170\end{array}$ \\
\hline
\end{tabular}

imation ( 1 ), but we reproduce remarkably well the relative triple splitting of the dipole plasmon peak. The two competing axial states in $\mathrm{Na}_{14}$ produce each a double-peaked structure where $\hbar \omega_{r}$ has double weight compared to $\hbar \omega_{z}$. The actual strength distribution is to be superposed incoherently from the two isomeric minima. One therefore expects four peaks or a washed-out structure if both minima have comparable occupation weight. But such quantitative details depend sensitively on the formation history of the clusters, and this is beyond our present approach.

In a local-current RPA $[10,18]$ it was shown that the coupling to volume plasmons (i.e., dipole modes involving local compression) lowers the frequencies of the surface plasmons by about $15-25 \%$ for light spherical clusters. This will persist for deformed clusters and lower the resonance energies. In deformed clusters, we also have to account for the coupling of dipole modes with modes of other multipolarities. Both kinds of couplings are presently being investigated and their effects in deformed clusters will be discussed elsewhere [7].
We thank O. Genzken for many inspiring discussions concerning this work and T. Hirschmann for the help in improving the accuracy of the $2 \mathrm{D}$ code. We acknowledge the Höchstleistungsrechenzentrum Jülich and the Leibniz Rechenzentrum München for the provided computing time.

\section{References}

[1] K. Selby, M. Vollmer, J. Masui, V. Kresin, W.A. de Heer and W.D. Knight, Phys. Rev. B 40 (1989) 5417

[2] K. Clemenger, Phys. Rev. B 32 (1985) 1359.

[3] W. Ekardt and Z. Penzar, Phys. Rev. B 38 (1988) 4273; Z. Penzar and W. Ekardt, Z. Phys. D, in press.

[4] D. Pines, Elementary excitations in solids (Benjamin, New York, 1981) ch. 3; G.D. Mahan, Many particle physics (Plenum, New York, $1981)$ ch. 5 .

[5] W. Ekardt, Phys. Rev. B 29 (1984) 1558.

[6] A. Rubio, L.C. Balbás and J.A. Alonso, in: 5th Int. Symp. on Small particles and inorganic clusters "ISSPIC5"; $\mathrm{Z}$ Phys. D (1991), in press.

[7] M. Brack, O. Genzken, G. Lauritsch and P.-G. Reinhard, to be published.

[8] D.E. Beck, Phys. Rev. B 30 (1984) 6935.

[9] W. Ekardt, Phys. Rev. B 31 (1985) 6360.

[10] M. Brack, Phys. Rev. B 39 (1989) 3533

[11] V. Blum, G. Lauritsch, J. Maruhn and P.-G. Reinhard, preprint (1990).

[12] G. Lauritsch and P.-G. Reinhard, in preparation.

[13] P.-G. Reinhard and R.Y. Cusson, Nucl. Phys. A 378 (1982) 418.

[14] D.L. Hill and J.A. Wheeler, Phys. Rev. 89 (1953) 1102

[15] D. Vautherin, Phys. Rev. C 7 (1973) 296.

[16] R. Englman, The Jahn-Teller effect in molecules and crystals (Wiley, New York, 1972)

[17] V. Bonacic-Koutecky, P. Fantucci and J. Koutecky, Phys. Rev. B 37 (1988) 4369, and earlier references quoted therein.

[18] P.-G. Reinhard, M. Brack and O. Genzken, Phys. Rev, A 41 (1990) 5568 